\title{
ON A THEOREM OF ABIKOFF
}

\section{LIPMAN BERS*}

This note contains a new proof and an extension of a theorem of Abikoff [1] on (complex) boundaries of Teichmüller spaces. First we recall some definitions and results, cf. [3] for references.

Let $G$ be a Fuchsian group operating on the upper half-plane $U$, i.e., a discrete subgroup of $\operatorname{PSL}(2, \boldsymbol{R})$. Let $B(L, G)$ be the complex Banach space of holomorphic functions $\varphi(z)$ defined in the lower half-plane $L$, with norm

$$
\|\varphi\|=\sup \left|y^{2} \varphi(z)\right|<\infty \quad(z=x+i y \in L),
$$

and satisfying the functional equation of quadratic differentials

$$
\varphi(g(z)) g^{\prime}(z)^{2}=\varphi(z), \quad g \in G .
$$

For every $\varphi \in B(L, G)$ the Schwarzian differential equation

$$
\{W, z\}=\left(\frac{W^{\prime \prime}(z)}{W^{\prime}(z)}\right)^{\prime}-\frac{1}{2}\left(\frac{W^{\prime \prime}(z)}{W^{\prime}(z)}\right)^{2}=\varphi(z)
$$

has meromorphic solutions in $L$; if $W$ is one, all others are of the form $\alpha W$ where $\alpha \in \operatorname{PSL}(2, \boldsymbol{C})$. It is convenient to denote by $W_{\varphi}$ the solution of (1) normalized by the requirement that

$$
W_{\varphi}(t-i)=\frac{1}{t}+O(t), \quad t \rightarrow 0
$$

Every $\varphi \in B(L, G)$ induces a homomorphism $\chi_{\varphi}$ of $G$ into $\operatorname{PSL}(2, C)$ defined by the rule

$$
W_{\varphi} \circ g(z)=\chi_{\varphi}(g) \circ W_{\varphi}(z) \quad(g \in G, z \in L) .
$$

(The group $G_{\varphi}=\chi_{\varphi}(G)$ is called the monodromy group of $\varphi$.)

The Teichmüller space $T(G)$ of $G$ can be defined as the set of those $\varphi \in B(L, G)$ for which $W_{\varphi}$ is the restriction to $L$ of a quasiconformal self-map $\hat{W}_{\varphi}$ of $\hat{\boldsymbol{C}}$, with

$$
\hat{W}_{\varphi} \circ g \circ \hat{W}_{\varphi}^{-1}(z)=\chi_{\varphi}(g)(z) \quad(g \in G, z \in \hat{C})
$$

(so that $G_{\varphi}$ is a quasi-Fuchsian group).

*) This material is based upon work partially supported by the National Science Foundation under Grant No. NSF MCS-78-27119. 
It is known that $T(G)$ is a domain (of holomorphy) in $B(L, G)$, containing the ball $\|\varphi\|<1 / 2$ and contained in the ball $\|\varphi\|<3 / 2$.

(We recall that $T(G)$ can be identified with the Teichmüller space $T\left(S_{G}\right)$ of the Riemann surface $S_{G}=U_{G} / G$ where $U_{G}$ is the complement in $U$ of all fixed points of elliptic elements of $U$. The dimension of $B(L, G)$ is finite if and only if $S_{G}$ is obtained from a closed surface of genus $p$ by removing $n$ distinct points, with $2 p-2+n$ $>0$; in this case $\operatorname{dim} T(G)=\operatorname{dim} B(L, G)=3 p-3+n$.)

From now we assume that $\operatorname{dim} B(L, G)>0$, i.e., that $G$ is not a triangle group, and denote by $\partial T(G)$ the boundary of $T(G)$ in $B(L, G)$. It is known and easy to show (cf. [3], p. 592) that for all points $\varphi \in \partial T(G)$ the map $W_{\varphi_{0}}$ is injective (so that $G_{\varphi_{0}}$ is discrete and has a non-empty region of discontinuity).

Theorem. Every neighborhood of every point $\varphi_{0} \in \partial T(G)$ contains points in the complement of $T(G) \cup \partial T(G)$.

For $\operatorname{dim} T(G)<\infty$ Abikoff [1] proved (by an ingenious use of plurisubharmonic functions) that $\varphi_{0} \in \partial T(G)$ has the property stated in the theorem whenever the limit set of $G_{\varphi_{0}}$ has measure 0 . He quotes an assertion by Thurston (the proof of which is not yet published) which implies that this is always so. Abikoff also notes that for $G=1$, the trivial group, the theorem follows at once from Gehring's characterization of the universal Teichmüller space $T(1)$ as the interior in $B(L, 1)$ of the set of Schwarzian derivatives of univalent functions [5].

The proof below (for all $G$ ) is based on an improvement of the "improved $\lambda$ lemma" of Sullivan - Thurston [6], which appears as Theorem 3 in Bers - Royden [4].

Assume that theorem is false for some $G$ and some $\varphi_{0} \in \partial T(G)$. Then there is an $\varepsilon>0$ such that for $\left\|\varphi-\varphi_{0}\right\|<\varepsilon$ the point $\varphi \in B(L, G)$ belongs to $T(G) \cup \partial T(G)$, so that $W_{\varphi}$ is injective.

Set

$$
E=W_{\varphi_{0}}(L)
$$

Choose a $\varphi_{1} \in T(G)$ with $\left\|\varphi_{1}-\varphi_{0}\right\|<\varepsilon / 4$ and set

and

$$
\dot{\psi}_{\lambda}=(1-4 \lambda) \varphi_{0}+4 \lambda \varphi_{1} \quad(|\lambda|<1)
$$

$$
f(\lambda, z)=W_{\psi_{\lambda}} \circ W_{\varphi_{0}}^{-1}(z) \quad(|\lambda|<1, z \in E) .
$$

Let $\Delta_{a}$ denote the disc $|\lambda|<a$ in $\boldsymbol{C}$.

The map $f: \Delta_{1} \times E \rightarrow \hat{C}$ is an injection of $E$ for every fixed $\lambda$, since

$$
\left\|\psi_{\lambda}-\varphi_{0}\right\|<\varepsilon \text { for }|\lambda|<1 \text {, }
$$

reduces to the identity for $\lambda=0$, since $\psi_{0}=\varphi_{0}$, and is holomorphic in $\lambda$ for every fixed $z \in E$ (with $f(\lambda, \infty)=\infty$ for all $\lambda$ ), since the solution of the Schwarzian differential equation (1) with $\varphi=\psi_{\lambda}$, satisfying the initial condition (2), depends holomorphically on $\lambda$. (To see this, recall that $W_{\varphi}$ admits the representation $W_{\varphi}=\eta_{1} / \eta_{2}$ 
where $\eta_{1}$ and $\eta_{2}$ are solutions of the linear differential equation $2 \eta^{\prime \prime}+\varphi \eta=0$ with $\eta_{1}=\eta_{2}^{\prime}=1$ and $\eta_{1}^{\prime}=\eta_{2}=0$ at $z=-i$.)

Applying Theorem 3 of [4] we conclude that there exists a unique map $\hat{f}$ : $\Delta_{1 / 3} \times \hat{\boldsymbol{C}} \rightarrow \hat{\boldsymbol{C}}$ with the following properties:

(j) For every fixed $\lambda,|\lambda|<1 / 3, \hat{f}(\lambda, \cdot)$ is a quasiconformal self-mapping of $\hat{\boldsymbol{C}}$, depending holomorphically on $\lambda$ and reducing to the identity for $\lambda=0$,

(jj) in every component $\omega$ of the complement $H$ of the closure $\hat{E}$ of $E$ the Beltrami coefficient of $f(\lambda, \cdot)$ is "harmonic" and depends holomorphically on $\lambda$,

(jjj) we have thai

$$
\hat{f}(\lambda, z)=f(\lambda, z) \text { for }|\lambda|<1 / 3 \text { and } z \in E .
$$

Condition (jj) means that

$$
\mu_{\omega}(\lambda, z)=\left(\frac{\partial \hat{f}(\lambda, z)}{\partial \bar{z}} \mid \frac{\partial \hat{f}(\lambda, z)}{\partial z}\right) \mid \omega=\varrho_{\omega}(z)^{-2} \overline{F_{\omega}(\lambda, z)}
$$

where $\varrho_{\omega}(z)|d z|$ is the Poincaré metric in $\omega$ and $F_{\omega}(\lambda, z)$ a function holomorphic in $z \in \omega$, antiholomorphic in $\lambda \in \Delta_{1 / 3}$. (If $H=\varphi$, (jj) is vacuous and the argument simplifies by itself.)

Now let $g \in G$ and set $g_{\lambda}=\chi_{\psi_{\lambda}}(g)$. Then

$$
g_{\lambda}(z)=W_{\psi_{\lambda}} \circ g \circ W_{\psi_{\lambda}}^{-1}(z) \text { for }|\lambda|<1 \text { and } z \in L
$$

since $W_{\psi_{\lambda}}$ is injective. Also $g_{0}=\chi_{\varphi_{0}}(g)$ since $\psi_{0}=\varphi_{0}$.

Consider the map $h: \Delta_{1 / 3} \times \hat{\boldsymbol{C}} \rightarrow \hat{\boldsymbol{C}}$ defined as

$$
h(\lambda, z)=g_{\lambda}^{-1} \circ \hat{f}\left(\lambda, g_{0}(z)\right) .
$$

It is holomorphic in $\lambda,|\lambda|<1 / 3$, for each fixed $z$, and is a quasiconformal selfmapping of $\hat{\boldsymbol{C}}$ for each fixed $\lambda$. Also,

$$
h(\lambda, z)=f(\lambda, z) \text { for }|\lambda|<1 / 3, \quad z \in E .
$$

Indeed, $g_{0}(E)=E$ and for $z \in E$ one has

$$
\begin{gathered}
\hat{f}\left(\lambda, g_{0}(z)\right)=f\left(\lambda, g_{0}(z)\right)=W_{\psi_{\lambda}} \circ W_{\varphi_{0}}^{-1} \circ W_{\varphi_{0}} \circ g \circ W_{\varphi_{0}}^{-1}(z) \\
=W_{\psi_{\lambda}} \circ g \circ W_{\varphi_{0}}^{-1}(z)=g_{\lambda} \circ W_{\psi_{\lambda}} \circ W_{\varphi_{0}}^{-1}(z)=g_{\lambda} \circ f(\lambda, z)
\end{gathered}
$$

whence (5) follows.

If $\omega$ is a component of the complement $H$ of $E$, so is $g_{0}(\omega)$, and a direct calculation based on (3) and (4) shows that for $z \in \omega$ the Beltrami coefficient of $h(\lambda, z)$ equals

$$
\begin{gathered}
\left(\frac{\partial h(\lambda, z)}{\partial \bar{z}} \mid \frac{\partial h(\lambda, z)}{\partial z}\right) \mid \omega=\mu_{g(\omega)}\left(\lambda, g_{0}(z)\right) \frac{\left|g_{0}^{\prime}(z)\right|^{2}}{g_{0}^{\prime}(z)^{2}} \\
=\left[\varrho_{g(\omega)}\left(g_{0}(z)\right) \mid g^{\prime}\left(z_{0}\right)\right]^{-2} \overline{F_{g(\omega)}\left(\lambda, g_{0}(z)\right) g_{0}^{\prime}(z)^{2}} \\
=\varrho_{\omega}(z)^{-2} \overline{F_{g(\omega)}\left(\lambda, g_{0}(z)\right) g_{0}^{\prime}(z)^{2}} .
\end{gathered}
$$


Since the function under the conjugation bar is holomorphic in $z$ and antiholomorphic in $\lambda$, and $\omega$ was arbitrary, we conclude that $h(\lambda, z)$ has a "harmonic" Beltrami coefficient in every component of the complement of $\hat{E}$. By the uniqueness part of Theorem 3 in [4] we obtain that

$$
h=\hat{f}
$$

and this shows that the restriction $\mu_{H}$ of the Beltrami coefficient of $\hat{f}$ to the complement $H$ of $\hat{E}$,

satisfies the relation

$$
\mu_{H}(\lambda, z)=\left(\frac{\partial \hat{f}(\lambda, z)}{\partial \bar{z}} / \frac{\partial \hat{f}(\lambda, z)}{\partial z}\right) \mid H,
$$

$$
\mu_{H}\left(\lambda, g_{0}(z)\right) \frac{\left|g_{0}^{\prime}(z)\right|^{2}}{\left(g_{0}^{\prime}(z)\right)^{2}}=\mu_{H}(\lambda, z) .
$$

Since $g \in G$ was arbitrary, this relation holds for all such $g$.

It follows from (7) that, for every $g \in G$ and every $\lambda \in \Delta_{1 / 3}$, the Beltrami coefficient of $\hat{f}(\lambda, \cdot) \circ g_{0} \mid H$ equals to $\mu_{H}(\lambda, \cdot)$ so that there exists a holomorphic function $\gamma(z), z \in H$, depending on $g$ and $\lambda$, such that

or

$$
\hat{f}(\lambda, \cdot) \circ g_{0} \mid H=\gamma \circ \hat{f}(\lambda, \cdot) H
$$

$$
\gamma=\hat{f}(\lambda, \cdot) \circ g_{0} \circ \hat{f}(\lambda, \cdot)^{-1} \mid H .
$$

This relation shows that $\gamma$ has a continuous extension to the boundary $\partial H$ of $H$, which is also the boundary $\partial E$ of $E$. But for $z \in E$

$$
\begin{aligned}
& \hat{f}(\lambda, \cdot) \circ g_{0} \circ \hat{f}(\lambda, \cdot)^{-1}(z)=f(\lambda, \cdot) \circ g_{0} \circ f(\lambda, \cdot)^{-1}(z) \\
& =W_{\psi_{\lambda}} \circ W_{\varphi_{0}}^{-1} \circ W_{\varphi_{0}} \circ g \circ W_{\varphi_{0}}^{-1} \circ W_{\varphi_{0}} \circ W_{\psi_{\lambda}}^{-1}(z)=g_{\lambda}(z) .
\end{aligned}
$$

Hence the boundary values of $\gamma$ in $H$ coincide with the values of $g_{\lambda}$ on $\partial H$. Since $\gamma$ and $g_{\lambda}$ are both holomorphic in $H, \gamma=g_{\lambda}$. We established that

$$
\hat{f}(\lambda, \cdot) \circ g \circ \hat{f}(\lambda, \cdot)^{-1}=g_{\lambda} .
$$

Since $\psi_{1 / 4}=\varphi_{1} \in T(G)$, the map $W_{\psi_{1 / 4}}$ has a quasiconformal extension $\hat{W}_{\psi_{1 / 4}}$ to $\hat{\boldsymbol{C}}$ such that

$$
\hat{W}_{\psi_{1 / 4}} \circ g \circ \hat{W}_{\psi_{1 / 4}}^{-1}=\chi_{\psi_{1 / 4}}(g) \quad(g \in G) .
$$

One checks at once that the map

is a quasiconformal extension of $W_{\varphi_{0}}$ to $\hat{\boldsymbol{C}}$ such that

$$
\hat{W}_{\varphi_{0}}=\hat{f}(1 / 4, \cdot)^{-1} \circ \hat{W}_{\psi_{1 / 4}}
$$

$$
\hat{W}_{\varphi_{0}} \circ g \circ \hat{W}_{\varphi_{0}}^{-1}=\chi_{\varphi_{0}}(g) \quad(g \in G) .
$$

This shows that $\varphi_{0} \in T(G)$. Contradiction.

Postscript. Abikoff observed that the proof above could be somewhat simplified by noting that (under the assumption made about $\varphi_{0}, \varepsilon$ and $\varphi_{1}$ ) the domain $E=$ 
$W_{\varphi_{0}}(L)$ is a Jordan domain, being the image of the Jordan domain $W_{\varphi_{1}}(L)$ under the quasiconformal self-map $f(1 / 4, \circ)^{-1}$ of $C$.

We note that this conclusion could be obtained already from the original "improved $\lambda$-lemma" by Sullivan and Thurston [6] without using the uniqueness statement in [4], and that it establishes the theorem for the case $\operatorname{dim} T(G)<\infty$. The uniqueness statement seems to be essential for treating the case $\operatorname{dim} T(G)=\infty$.

P.P.S. After this note was written I learned about Žuravlev's paper [7]. The main result of this paper asserts that $T(G)$ is the component (containing the origin) of the interior (in $B(L, G)$ ) of the set of Schwarzian derivatives (belonging to $B(L, G)$ ) of univalent functions. This assertion implies the theorem discussed here.

\section{References}

[1] AвIKoff, W.: A geometric property of Bers' embedding of the Teichmüller space. - Riemann surfaces and related topics: Proceedings of the 1978 Stony Brook Conference (State Univ. New York, Stony Brook, N. Y., 1978). Ann. of Math. Stud. 97, Princeton Univ. Press, Princeton, N. J., 1981, 3-5.

[2] Bers, L.: On boundaries of Teichmüller spaces and on Kleinian groups. I. - Ann. of Math. $91,1970,570-600$.

[3] Bers, L.: Finite-dimensional Teichmüller spaces and generalizations. - Bull. Amer. Math. Soc. (N. S.) 5, 1981, 131-172.

[4] Bers, L., and H. L. Royden: Holomorphic families of injections. - To appear.

[5] Gehring, F. W.: Univalent functions and the Schwarzian derivative. - Comment. Math. Helv. 52, 1977, 561-572.

[6] Sullivan, D., and W. P. Thurston: To appear.

[7] Žuravlev, I. V.: Univalent functions and Teichmüller spaces. - Soviet Math. Dokl. 21, 1980, 252-255; translated from Dokl. Akad. Nauk SSSR 250, 1980, 1047-1050.

Columbia University

Department of Mathematics

New York, NY 10027

USA

Received 28 October 1983 